\title{
Optimal Sensors Placement in Dynamic Damage Detection of Beams Using a Statistical Approach
}

\author{
Egidio Lofrano ${ }^{1}\left([) \cdot\right.$ Marco Pingaro $^{1}(1) \cdot$ Patrizia Trovalusci $^{1}([) \cdot$ \\ Achille Paolone ${ }^{1}$
}

Received: 9 June 2020 / Accepted: 26 September 2020 / Published online: 16 October 2020

(c) The Author(s) 2020

\begin{abstract}
Structural monitoring plays a central role in civil engineering; in particular, optimal sensor positioning is essential for correct monitoring both in terms of usable data and for optimizing the cost of the setup sensors. In this context, we focus our attention on the identification of the dynamic response of beam-like structures with uncertain damages. In particular, the non-localized damage is described using a Gaussian distributed random damage parameter. Furthermore, a procedure for selecting an optimal number of sensor placements has been presented based on the comparison among the probability of damage occurrence and the probability to detect the damage, where the former can be evaluated from the known distribution of the random parameter, whereas the latter is evaluated exploiting the closed-form asymptotic solution provided by a perturbation approach. The presented case study shows the capability and reliability of the proposed procedure for detecting the minimum number of sensors such that the monitoring accuracy (estimated by an error function measuring the differences among the two probabilities) is not greater than a control small value.
\end{abstract}

Keywords Sensors placement · Perturbation approach · Damage detection · Uncertain stiffness - Vibrating beam

Mathematics Subject Classification 47A55 - 70L05 · 74A45 · 74E35 · 74H10 · 74K10

\footnotetext{
$凶$ Egidio Lofrano

egidio.lofrano@uniroma1.it

Marco Pingaro

marco.pingaro@uniroma1.it

Patrizia Trovalusci

patrizia.trovalusci@uniroma1.it

Achille Paolone

achille.paolone@uniroma1.it

1 Sapienza University of Rome, Rome, Italy 


\section{Introduction}

Strategic constructions, such as bridges and tower buildings, during their lifetime are inexorably subjected to degradation and damage of various kinds. For this reason, careful monitoring is required for profitable maintenance and for activation of safety protocols in case of danger ("early warning" systems). The main problem, that discourages the use of large-scale structural health monitoring (SHM) system, is its high cost, directly related to the cost of the instrumentation and in particular the number of sensors used.

In this framework, dynamic approaches-mainly concerned with output-only techniques - have been successfully adopted to locate and quantify structural damages for historical [1,2] and recent construction [3,4]. Other methods have been proposed for the damage detection such as data-driven [5,6] and statistical method [7].

As reported by many authors, when structural responses of dynamic systems are exploited to trace the signature of the damage, one deals with an inverse problem, where damage influence is recognized starting from its effect. On the one hand, techniques not model based, see for instance [8,9], are able to directly detect the alterations due to any variation, but their mechanical interpretation is often cumbersome. On the other hand, model-based approaches, such as the techniques based on one-dimensional elements $[10,11]$, usually need to rely on accurate structural modeling and to select proper response signals. Damage effects have been also investigated, using non-classical continuum approaches, developed for materials with microstructure, by investigating displacement fields and wave propagation [12-14].

Evidently, a well position of the sensor network defines the suitability of dynamic behavior of the analyzed structure.

Therefore, a correct treatment that aims at reducing the number of sensors by limiting the cost of SHM without compromising the quality of monitoring is desirable. For this reason, optimal sensor placement (OSP) is an opening challenge in engineering $[15,16]$ and many different strategies have been proposed by several authors. The review paper [17] reports a comprehensive overview of a number of criteria for evaluating different sensor setups such as modal assurance criterion (MAC), the information entropy (IE) [18-21], singular value decomposition ratio (SVDR) [22], Fisher information matrix (FIM) [23,24], system-realization method [25] and multi-setup modal analysis [26]. A Bayesian framework for model-based optimal sensor placement for response predictions has been presented in [27,28]. A comparison of several methods for optimal sensors placement has been proposed in [29] applied to a real case study of a suspended bridge. In a recent work [30] a study of optimal sensor placement for timber frame structure has been developed. In [31] the conditions for the invertibility of linear system models have been discussed. In these methods, an optimization process has been performed to minimize an objective function. In order to solve the optimization process, different methodologies have been adopted such as improved genetic algorithms [32,33], and discrete optimization [34].

In the above-mentioned works, attention is paid to experimental modal analysis; however, there are few works on the identifiability of damage processes suffered by a structure. The recent work [35] focused on searching the weakest part of the structure in a random context. In this work, non-localized damages are adopted, which are in 
general more difficult to identify than narrow damages [36,37]. Damages are modeled as a perturbation of the healthy system and therefore a perturbation approach can properly describe the effect of the damage, both for standard [38] and state-space dynamic analysis [39,40]. In [41], a perturbation method has been proposed by some of the authors to derive the asymptotic eigensolution of a vibrating beam with uncertain damage. Then, the statistics of the random damage parameter have been obtained by means of an objective function minimizing the difference among analytical and experimental fractiles of the eigenvalues. The results obtained by the authors show the importance of considering the second order terms of the perturbation approach in order to achieve a significant increase in the identifiability of stochastic damages.

Starting from this model, we here propose a technique for obtaining an optimized solution of sensors placement. The proposed approach aims at exploiting the closedform asymptotic solution of the inverse problem to compare more combinations of number and placement of the sensors. These comparisons rely on an error function measuring the deviation from the analytical probability of damage occurrence. Due to the asymptotic nature of the research pattern, the performance of the proposed placement optimization technique has a low computational effort. Some preliminary results have been shown in [42]; the peculiarities and the capabilities of the technique are here deepened.

The main novelty of the technique, with respect to the cited bibliography, concerns the closed form evaluation of the identifiability of uncertain (local or widespread) damages. Even if the technique has been so far developed only for vibrating beams, the methodology is generally suitable for all cases when the dynamics of a structural system can be described through analytical solutions.

The paper is organized as follow: In Sect. 2, the details of the proposed optimal sensor placements have been presented. In Sect. 3, a numerical example, exploiting the capabilities of the proposed method, has been shown. Finally, in Sect. 4, some final remarks and future developments are reported.

\section{Proposed Procedure}

\subsection{Beam and Damage Model}

We consider in this work the classical beam model of the Euler-Bernoulli in which the shear deformation effect is assumed negligible. The governing equation of the free flexural undamped vibration reads:

$$
\frac{\partial^{2}}{\partial x^{2}}\left(B(x, \varepsilon) \frac{\partial^{2} v}{\partial x^{2}}\right)+\rho(x, \varepsilon) \frac{\partial v^{2}}{\partial t^{2}}=0 \quad x \in[0, L],
$$

where $B$ is the flexural stiffness and $\rho=\rho_{V} A$ is the mass density per unit length ( $\rho_{V}$ and $A$ being the volume mass density of the material and the area of the cross section, respectively). $x$ is the abscissa along the beam, of length $L, v$ is the transversal displacement, and $\varepsilon$ is a random variable related to damage (Fig. 1). In addition, the following assumptions were considered. 


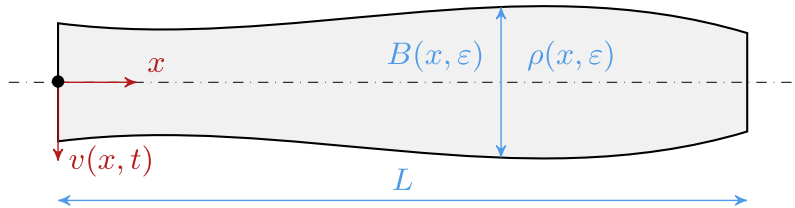

Fig. 1 Sketch of the beam model

1. Negligible changes of the mass, i.e., $\rho(x, \varepsilon)=\rho$. That is a fairly common hypothesis when one deals with damage identification.

2. Gaussian distribution of the random parameter $\varepsilon$ with mean value $\mu_{\varepsilon}$ and standard deviation $\sigma_{\varepsilon}$, i.e., $\varepsilon \sim \mathcal{N}\left(\mu_{\varepsilon}, \sigma_{\varepsilon}\right)$. The random parameter $\varepsilon$ defines the damage state of the beam and the related damage probability $P_{d}$ can be evaluated as:

$$
P_{d}=P(\varepsilon>0)=\int_{0}^{\infty} f(\varepsilon) d \varepsilon
$$

where $f(\varepsilon)$ is the probability density function of $\varepsilon$. In detail, since the random parameter $\varepsilon$ has been assumed Gaussian distributed, the probability of damage $P_{d}$ depends only on the ratio among the standard deviation $\sigma_{\varepsilon}$ and the mean value $\mu_{\varepsilon}$, that is, $P_{d}$ is a function of the so-called coefficient of variation $C V_{\varepsilon}=\sigma_{\varepsilon} / \mu_{\varepsilon}$.

3. Linear decomposition of the flexural stiffness as follow:

$$
B(x, \varepsilon)=B_{0}+\varepsilon B_{1}(x)=B_{0}-\varepsilon \underbrace{B_{0} e^{-\frac{\left(x-\mu_{b}\right)^{2}}{2 \sigma_{b}^{2}}}}_{B_{1}(x)}, \quad|\varepsilon| \ll 1,
$$

where $\mu_{b}$ characterizes the position around which the damage is centred and $\sigma_{b}$ is the relevant extension. The 'small' perturbation parameter $\varepsilon$ linearly scales the stiffness variation $B_{1}(x)$ of an initial (uniform) bending stiffness $B_{0}$. In our applications we pose a negative function $B_{1}(x)$, so that the random parameter $\varepsilon$ can be regarded as a random damage, where $\varepsilon=0$ implies an healthy beam, whereas a positive value of the parameter indicates a damage state (the higher the parameter, the greater the damage). We remark that $B_{1}(x)$ is a function establishing where the damage is located and how wide it is, and that the resolutive equations shown below are still true even for functions other than the bell-shaped one proposed here.

These positions reduce the entirety of the problem, they provide a useful simplification, allowing for a proper description of stochastic reductions (damage) and increases (reinforcement) of the beam stiffness [42].

With these assumptions and expanding all the terms in Eq. (1) one gets:

$$
\varepsilon \frac{\partial^{2} B_{1}(x)}{\partial x^{2}} \frac{\partial^{2} v}{\partial x^{2}}+2 \varepsilon \frac{\partial B_{1}(x)}{\partial x} \frac{\partial^{3} v}{\partial x^{3}}+\left(B_{0}+\varepsilon B_{1}(x)\right) \frac{\partial^{4} v}{\partial x^{4}}+\rho \frac{\partial^{2} v}{\partial t^{2}} .
$$


For the sake of simplicity, we adopt the following notation for the spatial $(\bullet)^{\prime}=$ $\partial(\bullet) / \partial x$ and time $(\bullet)=\partial(\bullet) / \partial t$ derivatives, respectively.

Introducing of the modal projection:

$$
v(x, t, \varepsilon)=\sum_{i=1}^{\inf } \phi(x, \varepsilon) q_{i}(t, \varepsilon),
$$

where $q_{i}(t, \varepsilon)=C_{i} \sin \left(\omega_{i}(\varepsilon) t+\theta_{i}\right)$ and $\phi_{i}(x, \varepsilon)$ are the mode shapes (eigenfunctions) and $\omega_{i}(\varepsilon)$ the relevant (circular) frequencies related to the eigenvalues $\lambda_{i}: \omega_{i}(\varepsilon)=$ $\lambda_{i}^{2}(\varepsilon) \sqrt{B_{0} / \rho}$. The governing equation in the modal space reads:

$$
\begin{aligned}
\omega_{i}^{2}(\varepsilon) & =-\frac{\ddot{q}_{i}(t, \varepsilon)}{q_{i}(t, \varepsilon)} \\
& =\frac{\varepsilon B_{1}^{\prime \prime}(x) \phi_{i}^{\prime \prime}(x, \varepsilon)+2 \varepsilon B_{1}^{\prime}(x) \phi_{i}^{\prime \prime \prime}(x, \varepsilon)+\left(B_{0}+\varepsilon B_{1}(x)\right) \phi_{i}^{\prime \prime \prime \prime}(x, \varepsilon)}{\rho \phi_{i}(x, \varepsilon)} .
\end{aligned}
$$

Since $\varepsilon$ is a small parameter, Eq. (6) can be solved through a perturbation approach. Moreover, the dynamic system under investigation is non-defective, and therefore, the eigensolution admits a perturbation series of integer powers of the small parameter:

$$
\begin{aligned}
& \lambda_{i}(\varepsilon)=\lambda_{0 i}+\varepsilon \lambda_{1 i}+\varepsilon^{2} \lambda_{2 i}+\ldots \\
& \phi_{i}(x, \varepsilon)=\phi_{0 i}(x)+\varepsilon \phi_{1 i}(x)+\varepsilon^{2} \phi_{2 i}(x)+\ldots
\end{aligned}
$$

Defining the operator $L[\bullet]=-B_{0} \lambda_{0}^{4}(\bullet)+B_{0}(\bullet)^{\prime \prime \prime \prime \prime}$ and balancing the same powers in $\varepsilon$, Eqs. (6) and (7) can be written as a series of equations as follows (where the dependence on $i$ has been omitted):

$$
\begin{aligned}
& \varepsilon^{0}: L\left[\phi_{0}(x)\right]=0 \\
& \varepsilon^{1}: L\left[\phi_{1}(x)\right]=4 B_{0} \lambda_{0}^{3} \lambda_{1} \phi_{0}(x)-B_{1}^{\prime \prime}(x) \phi_{0}^{\prime \prime}(x)+ \\
& \quad-2 B_{1}^{\prime}(x) \phi_{0}^{\prime \prime \prime}(x)-B_{1}(x) \phi_{0}^{\prime \prime \prime \prime}(x) \\
& \varepsilon^{2}: L\left[\phi_{2}(x)\right]=6 B_{0} \lambda_{0}^{2} \lambda_{1}^{2} \phi_{0}(x)+4 B_{0} \lambda_{0}^{3} \lambda_{2} \phi_{0}(x) \\
& \quad+4 B_{0} \lambda_{0}^{3} \lambda_{1} \phi_{1}(x)-B_{1}^{\prime \prime}(x) \phi_{1}^{\prime \prime}(x) \\
& \quad+2 B_{1}^{\prime}(x) \phi_{1}^{\prime \prime \prime}(x)+B_{1}(x) \phi_{1}^{\prime \prime \prime \prime}(x) \\
& \quad \ldots
\end{aligned}
$$

The solution of Eq. (8) $)_{1}$-so-called generating equation-is the well-known one:

$$
\phi_{0 i}(x)=C_{1} \cos \left(\lambda_{0 i} x\right)+C_{2} \sin \left(\lambda_{0 i} x\right)+C_{3} \cosh \left(\lambda_{0 i} x\right)+C_{4} \sinh \left(\lambda_{0 i} x\right) .
$$

The boundary conditions posed (BC) allow us to compute the eigenvalues $\lambda_{0 i}$ and three of the four constants $C_{j}, j=1, \ldots, 4$ (the fourth one is a generic constant scaling the mode). Once the pair $\left(\lambda_{0 i} ; \phi_{0 i}(x)\right)$ are known, Eq. $(8)_{2}$ gives $\left(\lambda_{1 i} ; \phi_{1 i}(x)\right)$, Eq. $(8)_{3}$ 
gives $\left(\lambda_{2 i} ; \phi_{2 i}(x)\right)$ and so on. After some algebra, the expressions of the eigenvalues and the eigenfunctions for the first and second order terms can be calculated:

$$
\begin{aligned}
\lambda_{1 i}= & \frac{\int_{0}^{L} \phi_{0 i}(x) B_{1}^{\prime \prime}(x) \phi_{0 i}^{\prime \prime}(x) d x+2 \int_{0}^{L} \phi_{0 i}(x) B_{1}^{\prime}(x) \phi_{0 i}^{\prime \prime \prime}(x) d x}{4 B_{0} \lambda_{0 i}^{3} \int_{0}^{L} \phi_{0 i}^{2}(x) d x} \\
& +\frac{\lambda_{0 i}^{4} \int_{0}^{L} B_{1}(x) \phi_{0 i}^{2}(x) d x}{4 B_{0} \lambda_{0 i}^{3} \int_{0}^{L} \phi_{0 i}^{2}(x) d x} \\
\lambda_{2 i}=\quad & \frac{-3 \lambda_{1 i}^{2} \int_{0}^{L} \phi_{0 i}^{2}(x) d x-2 \lambda_{0 i} \lambda_{1 i} \int_{0}^{L} \phi_{0 i}(x) \phi_{1 i}(x) d x}{2 \lambda_{0 i} \int_{0}^{L} \phi_{0 i}^{2}(x) d x} \\
& +\frac{\int_{0}^{L} \phi_{0 i}(x) B_{1}^{\prime \prime}(x) \phi_{1 i}^{\prime \prime}(x) d x+2 \int_{0}^{L} \phi_{0 i}(x) B_{1}^{\prime}(x) \phi_{1 i}^{\prime \prime \prime}(x) d x}{4 B_{0} \lambda_{0 i}^{3} \int_{0}^{L} \phi_{0 i}^{2}(x) d x} \\
& +\frac{\int_{0}^{L} \phi_{0 i}(x) B_{1}(x) \phi_{1 i}^{\prime \prime \prime \prime}(x) d x}{4 B_{0} \lambda_{0 i}^{3} \int_{0}^{L} \phi_{0 i}^{2}(x) d x}
\end{aligned}
$$

for the first and second order terms of the eigenvalues, and:

$$
\begin{aligned}
\phi_{1 i}(x)= & \alpha_{i i} \phi_{0 i}(x)+\sum_{\substack{k=1 \\
k \neq i}}^{\infty} \alpha_{i k} \phi_{0 k}(x) \\
\alpha_{i k}= & \frac{\int_{0}^{L} \phi_{0 k}(x) B_{1}^{\prime \prime}(x) \phi_{0 i}^{\prime \prime}(x) d x+2 \int_{0}^{L} \phi_{0 k}(x) B_{1}^{\prime}(x) \phi_{0 i}^{\prime \prime \prime}(x) d x}{B_{0}\left(\lambda_{0 i}^{4}-\lambda_{o k}^{4}\right) \int_{0}^{L} \phi_{0 k}^{2}(x) d x} \\
& +\frac{\lambda_{0 i}^{4} \int_{0}^{L} \phi_{0 k}(x) B_{1}(x) \phi_{0 i}(x) d x}{B_{0}\left(\lambda_{0 i}^{4}-\lambda_{o k}^{4}\right) \int_{0}^{L} \phi_{0 k}^{2}(x) d x} \\
\phi_{2 i}(x)= & \beta_{i i} \phi_{0 i}(x)+\sum_{\substack{k=1 \\
k \neq i}}^{\infty} \beta_{i k} \phi_{0 k}(x) \\
\beta_{i k}= & \frac{-4 B_{0} \lambda_{0 i}^{3} \lambda_{1 i} \int_{0}^{L} \phi_{0 k}(x) \phi_{1 i}(x) d x+\int_{0}^{L} \phi_{0 k}(x) B_{1}^{\prime \prime}(x) \phi_{1 i}^{\prime \prime}(x) d x}{B_{0}\left(\lambda_{0 i}^{4}-\lambda_{0 k}^{4}\right) \int_{0}^{L} \phi_{0 k}^{2}(x) d x} \\
& +\frac{2 \int_{0}^{L} \phi_{0 k}(x) B_{1}^{\prime}(x) \phi_{1 i}^{\prime \prime \prime}(x) d x+\int_{0}^{L} \phi_{0 k}(x) B_{1}(x) \phi_{1 i}^{\prime \prime \prime \prime}(x) d x}{B_{0}\left(\lambda_{0 i}^{4}-\lambda_{0 k}^{4}\right) \int_{0}^{L} \phi_{0 k}^{2}(x) d x}
\end{aligned}
$$

for the first- and second-order terms of the eigenfunctions.

\subsection{Optimal Sensor Placement (OSP)}

Let us focus on a beam defined by its stiffness $B(x, \varepsilon)$, linear mass density $\rho(x, \varepsilon)=\rho$ and length $L$, such as described in Sect. 2.1. The solution of Eq. (7) gives a closed- 
form expression of the eigenvalues and eigenfunctions here adopted to estimate the identifiability of damages in the vibrating uncertain beam. Moreover, the damage state of the beam is governed by the random parameter $\varepsilon$, that has been assumed gaussian distributed (see Sect. 2.1), and the probability of damage $P_{d}$, defined in Eq. (2) is a function of the coefficient of variation $C V_{\varepsilon}=\sigma_{\varepsilon} / \mu_{\varepsilon}$.

The probability to detect the damage resorting to a statistical dynamic analysis is related to the reduction of the eigenvalues:

$$
P_{d d i}=P\left(\lambda_{i}(\varepsilon)<\lambda_{0 i}\right)=P\left(\lambda_{i}(\varepsilon)-\lambda_{0 i}<0\right) \quad i=1, \ldots, n_{\lambda}
$$

being $n_{\lambda}$ the number of the first (measured) eigenvalues (related to sampling frequency of the sensors). Using Eq. (7) $)_{1}$, the differences $\lambda_{i}(\varepsilon)-\lambda_{0 i}$ can be written as: $\varepsilon \lambda_{1 i}+\left(\varepsilon / \sigma_{\varepsilon}\right)^{2} \sigma_{\varepsilon}^{2} \lambda_{2 i}$, where $\varepsilon$ is gaussian distributed, $\varepsilon \sim \mathcal{N}\left(\mu_{\varepsilon}, \sigma_{\varepsilon}\right)$, whereas the term $\left(\varepsilon / \sigma_{\varepsilon}\right)^{2}$ has a noncentral chi-squared distribution $\mathcal{X}^{2}\left(k_{\chi}, \lambda_{\chi}\right)$, with a unitary degree of freedom $k_{\chi}$ and non-centrality parameter $\lambda_{\chi}$ equals to $\left(\mu_{\varepsilon} / \sigma_{\varepsilon}\right)^{2}$. Therefore, the probability density function of the term $\lambda_{i}(\varepsilon)-\lambda_{0 i}$ is a mixture of two known distributions. For the sake of brevity, the relevant probability $P_{d d i}$ is here explicitly evaluated only in the case when the two derivatives $\lambda_{1 i}$ and $\lambda_{2 i}$ are both negative (which is the most usual case):

$$
P_{d d i}=\gamma_{1 i} w_{1 i} \frac{1}{2}\left(1+\operatorname{Erf}\left[\frac{1}{\sqrt{2} C V_{\varepsilon}}\right]+\operatorname{Erfc}\left[\frac{\lambda_{1 i}+\lambda_{2 i} \mu_{\varepsilon}}{\sqrt{2} C V_{\varepsilon} \lambda_{2 i} \mu_{\varepsilon}}\right]\right)+\gamma_{2 i} w_{2 i}
$$

with $i=1, \ldots, n_{\lambda}$ and where Erf and Erfc are the error function and the complementary error function, respectively. The two weights $w_{1 i}$ and $w_{2 i}$ can be expressed as:

$$
w_{1 i}=\frac{\left|\lambda_{1 i}\right|}{\left|\lambda_{1 i}\right|+\sigma_{\varepsilon}^{2}\left|\lambda_{2}\right|}, \quad w_{2 i}=\frac{\sigma_{\varepsilon}^{2}\left|\lambda_{2 i}\right|}{\left|\lambda_{1 i}\right|+\sigma_{\varepsilon}^{2}\left|\lambda_{2 i}\right|}
$$

The penalty indexes affecting the first and the second order term are: $\gamma_{1 i}=1-$ $\left|\tilde{A}_{1 i}-A_{1 i}\right| / A_{1 i}$ and $\gamma_{2 i}=1-\left|\tilde{A}_{2 i}-A_{2 i}\right| / A_{2 i}$, respectively. These two indexes are introduced to describe in a synthetic way the accuracy in the reconstruction of the variations encountered by the mode shapes. Several definitions can be adopted for this purpose. In this work, penalty is related to the discretization error of experimental mode shapes, i.e., $\gamma_{1 i}$ and $\gamma_{2 i}$ are evaluated comparing the continuous (analytical) mode shapes and the discretized (measurable by the network) ones. $A_{1 i}$ and $A_{2 i}$ are the analytical absolute values of the areas under the graph of $\left|\phi_{1 i}(x)\right|$ and $\left|\phi_{2 i}(x)\right|$, respectively, whereas $\tilde{A}_{1 i}$ and $\tilde{A}_{2 i}$ are the discretized counterparts:

$$
A_{1 i}=\int_{0}^{L}\left|\phi_{1 i}(x)\right| d x, \quad A_{2 i}=\int_{0}^{L}\left|\phi_{2 i}(x)\right| d x
$$




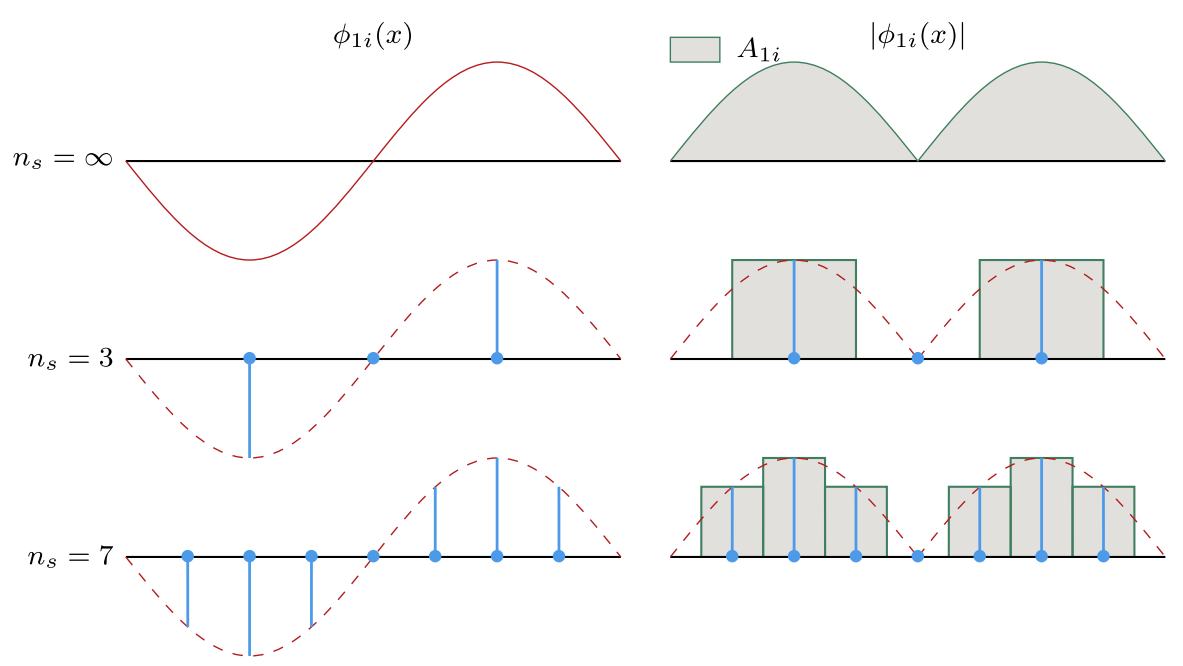

Fig. 2 Representation of $\left|\phi_{1 i}\left(x_{k}\right)\right|$ for different values of the number $n_{s}$ of sensors

and

$$
\begin{aligned}
& \tilde{A}_{1 i}=\sum_{s=1}^{n_{s}}\left|\phi_{1 i}\left(x_{k}\right)\right| \frac{x_{k+1}-x_{k-1}}{2}, \\
& \tilde{A}_{2 i}=\sum_{s=1}^{n_{s}}\left|\phi_{2 i}\left(x_{k}\right)\right| \frac{x_{k+1}-x_{k-1}}{2}, \quad \text { with: } x_{0}=0, x_{n_{s}+1}=L,
\end{aligned}
$$

where $x_{k}$ and $n_{s}$ are the positions of sensors along the beam axis and the number of sensors, respectively.

An example of the areas under the graph of $\left|\phi_{1 i}\left(x_{k}\right)\right|$ for different values of the number of sensors is reported in Fig. 2, which shows that $\gamma_{1 i}$ and $\gamma_{2 i}$ tend to 1 (which implies no penalty) when the number $n_{s}$ of the sensors tends to infinity.

The discrepancy between the probability of damage occurrence, $P_{d}$ defined in Eq. (2), and the probability to detect the damage, $P_{d d i}$ defined Eq. (13), measures the capabilities of the sensor network to detect the changes in eigenvalues and eigenfunctions due to structural damages (that is, the damage):

$$
\Delta P=\sum_{i=1}^{n_{\lambda}} \frac{1}{n_{\lambda}}\left(P_{d}-P_{d d i}\right) .
$$

Eq. (17) is a function of the number $n_{\lambda}$ of measured eigenvalues, the positions $x_{k}$ of sensors along the beam axis and the number $n_{s}$ of sensors, i.e., $\Delta P=\Delta P\left(n_{\lambda}, n_{s}, x_{k}\right)$.

Assuming that an optimal sensors placement is the one requiring less sensors, the best sensors network for a given number $n_{\lambda}$ of measured eigenvalues can be defined 
through the following constrained optimization problem, where the objective function is not-strictly convex and constraint is an "hard-like" nonlinear inequality:

determine $\min F\left(n_{S}\right)=n_{s}$ subjected to:

$$
\Delta P\left(n_{s}, x_{k}\right) \leq T o l,
$$

with the bounds on the unknowns:

$n_{s}>0$,

$x_{k} \geq 0, x_{k} \leq L, k=1, \ldots, n_{s}$

where $T o l=\overline{\Delta P}$ is a chosen threshold value for the difference among the probability of damage and the probability of detecting the damage. The proposed procedure is resumed in the flowchart of Fig. 3, where $E_{0}$ is the initial value of the Young's modulus and $I_{0}$ the initial value of the cross-sectional moment of inertia (so that the undamaged bending stiffness $B_{0}$ is equal to $\left.E_{0} I_{0}\right)$.

\section{Application}

\subsection{Case Study}

The case study is a simply supported beam, which geometry and material in the undamaged scenario (the input values of the flowchart) are: Young's modulus $E_{0}=$ $30 \cdot 10^{9} \mathrm{~Pa}$, volume mass density $\rho_{V}=2500 \mathrm{hboxkg} / \mathrm{m}^{3}$, length $L=6 \mathrm{~m}$ and rectangular cross section of base $0.30 \mathrm{~m}$ and height $0.60 \mathrm{~m}$ (hence the initial moment of inertia $I_{0}=0.0054 \mathrm{~m}^{4}$ ); see Fig. 4. Assuming these values, a linear mass density of $\rho=450 \mathrm{~kg} / \mathrm{m}$ and an initial bending stiffness of $B_{0}=E_{0} I_{0}=162 \cdot 10^{6} \mathrm{~N}$ $\mathrm{m}^{2}$ are found. We note that these values are quite typical for a 6-m-long concrete beam. Equation (9) plus the proper boundary conditions leads to the well-known eigensolution of the undamaged beam: $\lambda_{0 i}=i \pi / L$, for the eigenvalues, and $\phi_{0 i}=$ $\sin \left(\lambda_{0 i} x\right)$, for the (unitary scaled) mode shapes.

The stiffness variation, and therefore the damage as postulated in Sect. 2.1, is obtained using Eq. (3).

For the shape of the damage, that is, for $B_{1}(x)$, we assume that the damage is centred at the midspan of the beam, whence $\mu_{b}=L / 2$, but with three different values of the relevant extension $\sigma_{b}=\{L / 60, L / 30, L / 15\}$. For the random parameter $\varepsilon$, the mean value is set to $\mu_{\varepsilon}=0.20$ and three standard deviations are imposed $\sigma_{\varepsilon}=\{0.04,0.20,0.36\}$; these three values of the standard deviation $\sigma_{\varepsilon}$ correspond to the following three values of the coefficient of variation $C V_{\varepsilon}=\{0.2,1.0,1.8\}$.

With these values we intend to represent usual real-life cases: when one needs to set a sensor network to monitor the health of a structure (beam-like in our case), both the position in which the damage is likely to occur and its magnitude can be postulated or estimated quite easily (for instance, with nonlinear models in ultimate limit state conditions). From this standpoint, the central section is certainly the most prone to damage for a simply supported beam (hence the value L/2 for $\mu_{b}$ ); at the same time, a $20 \%$ reduction in stiffness $\left(\mu_{\varepsilon}\right.$ of 0.20$)$ is also a typical and reasonable value. 


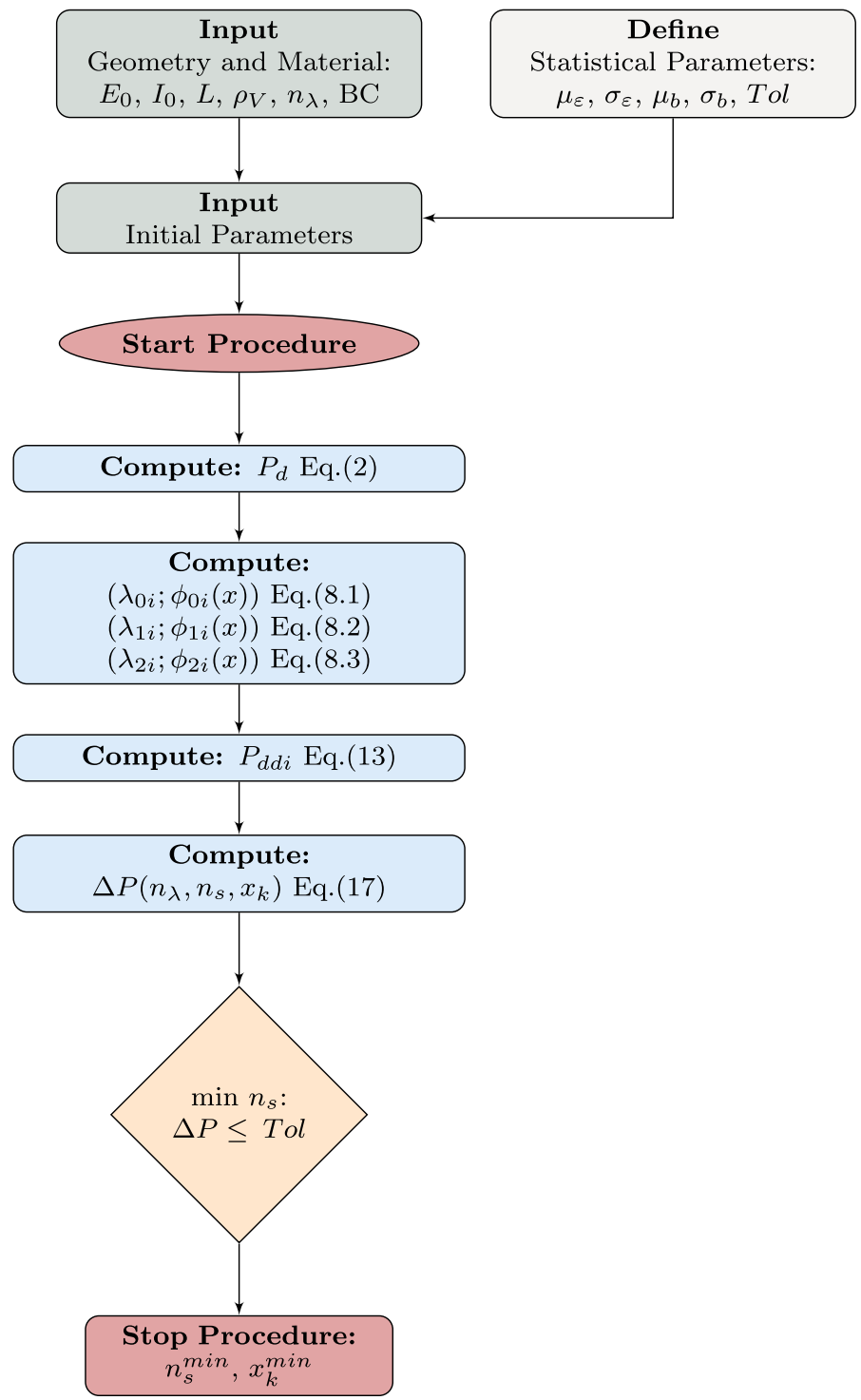

Fig. 3 Flow-chart of the proposed procedure

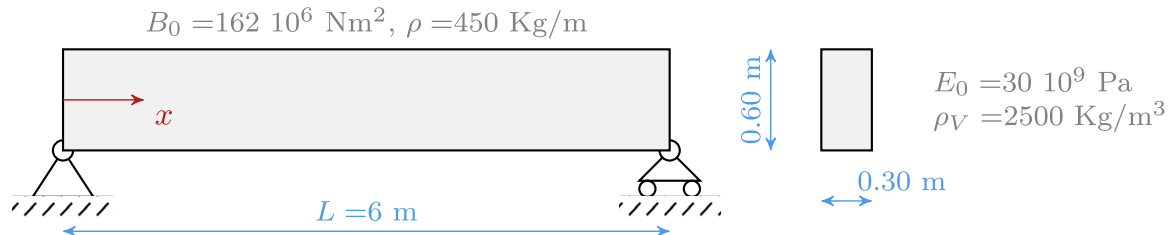

Fig. 4 Case study 


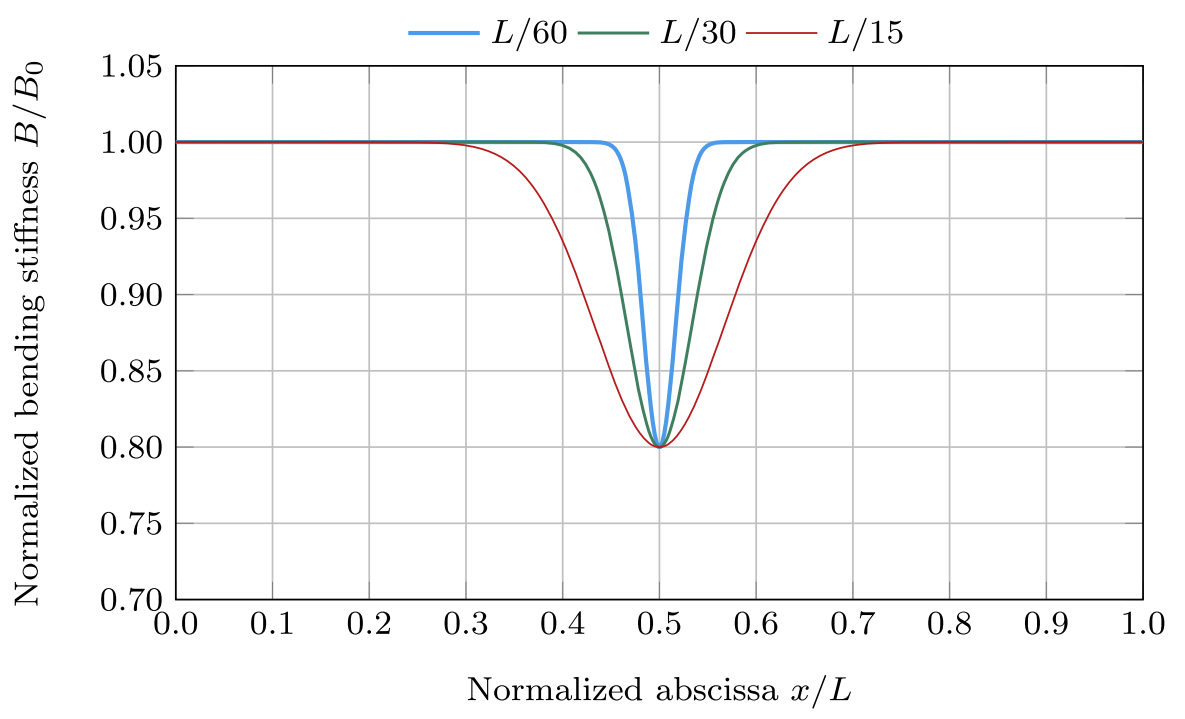

Fig. 5 Beam stiffness for a damage centred at midspan $\left(\mu_{b}=L / 2\right)$ with three different extensions $\sigma_{b}=$ $\{L / 60, L / 30, L / 15\}$

Conversely, it is difficult to model and calculate the spatial amplitude $\sigma_{b}$ of the damage and the scattering (uncertainty) $\sigma_{\varepsilon}$ of its magnitude. For this reason, here we assume a wide range of possible values for $\sigma_{b}$ (from $L / 60$ to $L / 15$ ) and $\sigma_{\varepsilon}$ (from 0.04 to 0.36$)$.

To give an idea of the damage that is being considered, Fig. 5 shows the trend of the bending stiffness $B(x, \varepsilon)$ with the variation of the spatial amplitude $\sigma_{b}$ (considering for the random parameter intensity $\varepsilon$ a value of 0.20 ), whereas in the case where $\sigma_{b}$ is equal to $\mathrm{L} / 60$, the damage is practically concentrated at the center section, for $\sigma_{b}$ equal to L/15 the damage extends for almost a third of the beam.

As regards the chosen values of the standard deviation $\sigma_{\varepsilon}$ of the random parameter $\varepsilon$, the relevant probability of damage $P_{d}$ is shown with the help of Fig. 6: when the coefficient of variation attains the values $C V_{\varepsilon}=\{0.2,1.0,1.8\}$, the probability of damage $P_{d}$ is equal to 100,84 , and $71 \%$, respectively. It clearly turns out that the first case can be assimilated to a deterministic damage.

To run the flowchart of Fig. 3, two other parameters must be set:

- the tolerance value $T o l$ for the probability discrepancy $\Delta P$. Here we use a value of $10 \%$, which seems to be a good compromise between the wish of prediction accuracy and the need to contain the number of sensors;

- the number of measured eigenvalues $n_{\lambda}$. Three values are here adopted, $n_{\lambda}=$ $\{2,4,8\}$, well representative of the number of eigenvalues typically measured in real beams. 


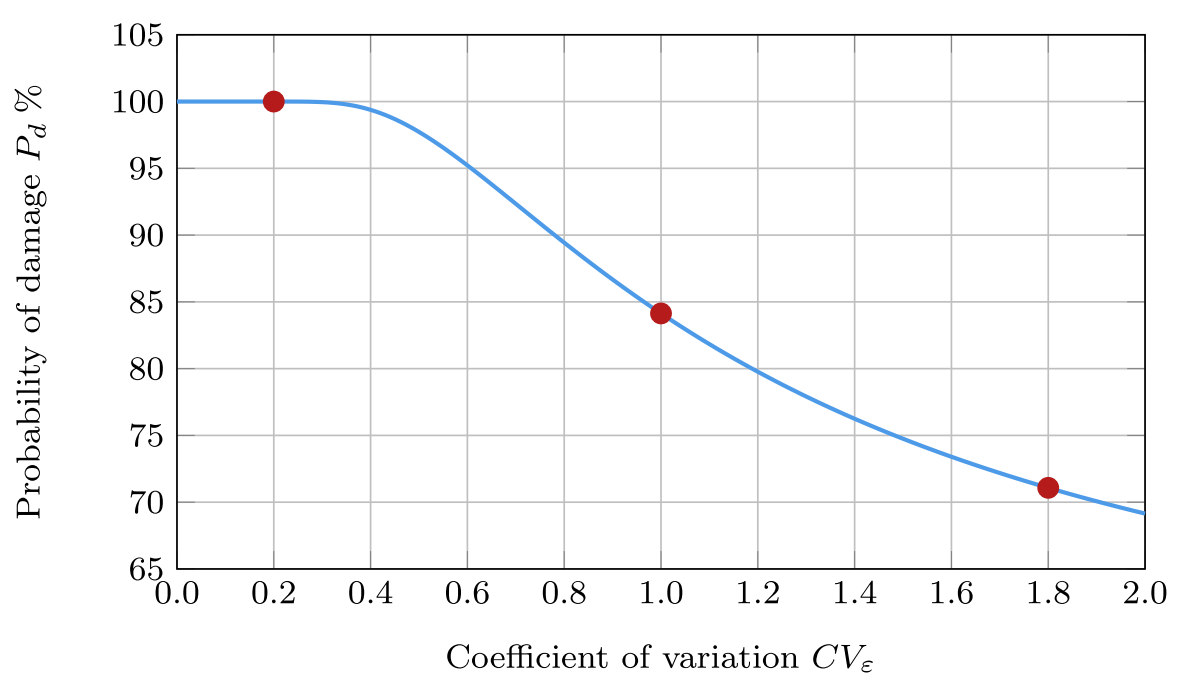

Fig. 6 Variation of the probability of damage $P_{d}$ with the coefficient of variation $C V_{\varepsilon}$; red dots are the values chosen for the application $\left(C V_{\varepsilon}=\{0.2,1.0,1.8\}\right)$

\subsection{Results}

In this section, we show the results of the constrained optimization problem in Eq. (18). Since three different values have been assumed for the coefficient of variation $C V_{\varepsilon}$, for the spatial amplitude of the damage $\sigma_{b}$ and for the number of measured eigenvalues $n_{\lambda}$, a total amount of 27 runs are here performed. As also shown in the flow-chart of Fig. 3, the results of the technique are provided in terms of the minimum number of sensors $n_{s}$ and their position $x_{k}, k=1, \ldots, n_{s}$ along the beam axis. To pose a computationally simpler problem, we assume hereafter that networks are composed of equally spaced sensors: under this hypothesis, the solution is just given as $n_{s}$, i.e., the minimum number of sensors.

With reference to the case of four measured eigensolutions $\left(n_{\lambda}=4\right)$ and coefficient of variation of $0.20\left(C V_{\varepsilon}=0.20\right)$, Fig. 7 shows the variation of the percentage probability difference $\Delta P$ (Eq. (17)) when $n_{s}$ ranges from 1 to 10 . When the number of sensors is increased, there is a "physically sound" reduction of the percentage difference; moreover, as even suggested by the mechanical meaning of the spatial amplitude $\sigma_{b}$, the reduction with $n_{s}$ is more pronounced when $\sigma_{b}$ is greater, that is, when the damaged area is increased. Recalling the adopted threshold Tol of $10 \%$, for all these three cases, we obtain as optimal (equally spaced) sensors placement the one considering eight sensors.

Table 1 shows the results obtained for the 27 runs of the constrained optimization problem. In detail, the table shows the minimum number of sensors $n_{s}$ needed to ensure a percentage probability difference $\Delta P$ less than the adopted threshold $T o l$ of $10 \%$. As a general comment, within the ranges of variation considered in this application, the minimum number of sensors $n_{s}$ changes from 6 to 10; the plots shown 


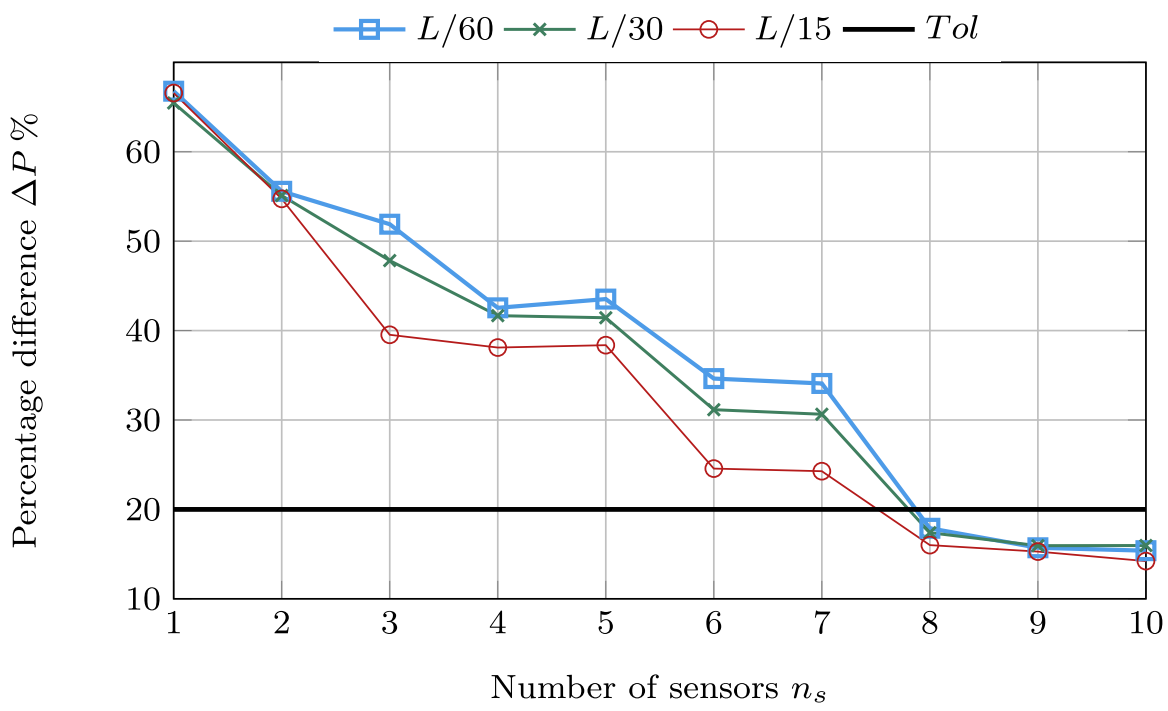

Fig. 7 Selection of the optimal number of sensors for the cases: four eigensolutions $\left(n_{\lambda}=4\right)$, coefficient of variation equal to $0.20\left(C V_{\varepsilon}=0.20\right)$ and three different spatial amplitudes $\sigma_{b}=\{L / 60, L / 30, L / 15\}$

in the previous Fig. 7 are related to the values highlighted in the table through a gray background. Some significant remarks can be collected:

- following the previous comments on Fig. 7, for a given number $n_{\lambda}$ of eigenvalues and a given coefficient of variation $C V_{\varepsilon}$, the minimum number $n_{s}$ tends to decrease if the damage is more widespread (i.e., when $\sigma_{b}$ is increased);

- for a given number $n_{\lambda}$ of eigenvalues and a given spatial amplitude $\sigma_{b}$ of the damage, the minimum number $n_{s}$ tends to decrease if the damage is more uncertain (i.e., when the coefficient of variation $C V_{\varepsilon}$ is increased). This evidence is to be connected to a simple circumstance: as the coefficient of variation $C V_{\varepsilon}$ increases, the probability of damage $P_{d}$ decreases, therefore, having imposed a constant tolerance $T$ ol (between the probability $P_{d}$ and the probability to detect the damage $\left.P_{d d i}\right)$, the minimum number $n_{s}$ must necessarily decrease;

- the last condition to be analyzed is the one revealed considering a given spatial amplitude $\sigma_{b}$ of the damage and a given coefficient of variation $C V_{\varepsilon}$ of the random parameter $\varepsilon$. The results show that when the number of eigenvalues $n_{\lambda}$ increases, also the required minimum number of sensors $n_{s}$ increases. At a first glance, this result may seem counterintuitive, because one would expect that as the number of information (the number of eigenvalues) of the single sensor increases, the network consequently needs fewer sensors to achieve a certain performance. In truth, the result here obtained is fully consistent with the proposed procedure. Indeed, our technique provides as criterion for selecting the minimum number of sensors $n_{s}$, the evaluation of a difference in probability which is an average value considering all the contributions of the measured eigenvalues (see Eq. (13) and Eq. (17)). Taking into account that these contributions of the individual eigensolution are 
Table 1 Optimal (minimum) number of sensors $n_{s}$ for different combination of: number of eigensolutions $n_{\lambda}$, coefficient of variation $C V_{\varepsilon}$ of the random parameter $\varepsilon$ and spatial amplitude $\sigma_{b}$ of the damage

\begin{tabular}{|c|c|c|c|c|}
\hline \multirow{2}{*}{$n_{s}$ min for } & \multicolumn{3}{|c|}{$C V_{\varepsilon}$} \\
\cline { 2 - 5 }$n_{\lambda}=\mathbf{2}$ & 0.2 & 1.0 & 1.8 \\
\hline \multirow{3}{*}{$\sigma_{b}$} & $\mathrm{~L} / 60$ & 8 & 8 & 8 \\
\cline { 2 - 5 } & $\mathrm{L} / 30$ & 8 & 6 & 6 \\
\cline { 2 - 5 } & $\mathrm{L} / 15$ & 6 & 6 & 6 \\
\hline
\end{tabular}

\begin{tabular}{|c|c|c|c|c|}
\hline \multirow{2}{*}{$n_{s}$ min for } & \multicolumn{3}{|c|}{$C V_{\varepsilon}$} \\
\cline { 5 - 5 }$n_{\lambda}=4$ & 0.2 & 1.0 & 1.8 \\
\hline \multirow{3}{*}{$\sigma_{b}$} & $\mathrm{~L} / 60$ & 8 & 8 & 8 \\
\cline { 2 - 2 } \cline { 5 - 5 } & $\mathrm{L} / 30$ & 8 & 8 & 8 \\
\cline { 5 - 5 } \cline { 5 - 5 } & $\mathrm{L} / 15$ & 8 & 8 & 6 \\
\hline
\end{tabular}

\begin{tabular}{|c|c|c|c|c|}
\hline \multirow{2}{*}{$n_{s}$ min for } & \multicolumn{3}{|c|}{$C V_{\varepsilon}$} \\
\cline { 2 - 5 }$n_{\lambda}=\mathbf{8}$ & 0.2 & 1.0 & 1.8 \\
\hline \multirow{3}{*}{$\sigma_{b}$} & $\mathrm{~L} / 60$ & 10 & 10 & 9 \\
\cline { 2 - 5 } & $\mathrm{L} / 30$ & 10 & 10 & 8 \\
\cline { 2 - 5 } & $\mathrm{L} / 15$ & 10 & 8 & 8 \\
\hline
\end{tabular}

weighted on the discretization error of the perturbed mode shapes (see the penalty indexes of Eq. (13)), it is evident that as the number of eigenvalues is increased, even the required minimum number of sensors $n_{s}$ increases (because it is required to describe higher, "more distorted", modes). In other words, to see a benefit linked to a greater number of measured eigenvalues, a different relation should be used for the difference in probability Eq. (17), for example, instead of the average value, the minimum value of the difference between the damage probability $P_{d}$ and the probability to detect the damage $P_{d d i}$ could be considered.

\section{Conclusions}

The main goal of this paper was the damage identification in transversely vibrating beams with uncertain stiffness. The beam model is non-deformable for any shear deformation and changes in mass due to structural damages are assumed negligible. Damage is then introduced as a "small" perturbation of the uniform initial bending stiffness. In detail, the damaged scenario is described with two terms: a bell-shaped function, ruling the position around which the damage is centred and its extension; and the "small" random parameter, which controls the intensity of the damage. Then, the adoption of a modal projection in conjunction with a perturbation technique allows us to achieve the eigensolutions in closed-form.

Moving towards the sensor network, the hypothesis of Gaussian distribution of the damage random parameter leads to a robust procedure for an optimal placement of 
sensors. It is based on the comparison among the probability of damage occurrence, evaluated from the known distribution of the random parameter, and the probability to detect the damage, evaluated exploiting the closed-form asymptotic solution described above. This comparison brings us to a constrained optimization problem, where the constraint is ruled by an error function encompassing three parameters: the number of measured eigenvalues, the positions of the sensors along the beam axis and the number of sensors.

A simple and paradigmatic case study, whose behavior is well known and clear, was considered to highlight the advantages and disadvantages of the proposed technique. The parametric investigation of a simply supported beam with rectangular cross section showed how the proposed approach is a suitable tool to collect and compare the performance of different sensors configurations. On the other hand, the main limitation of the proposed technique is the adoption of analytical solutions. This approach excludes its generalization to structures more general or complex than beam-like, for which closed form solutions are not pervasive.

Starting from the procedure here developed, there are some future developments capable of improving and generalizing the procedure itself. In particular:

- here the optimization refers to a given, expected, damaged scenario, but more plausible damage models would be desirable;

- a penalty index taking into account the sensitivity of the sensors should be introduced to describe in the procedure the lower frequency shift detectable by the sensors;

- the definition used to measure the accuracy of the sensor networks, based on an average difference (Eq. (17)), could be "weaken" or "strengthen" (looking at the lower or at the greater difference of the summation); the relevant effects on the optimal sensors placements are being analyzed;

- an extension of the proposed methodology for 2D continua is under investigation in order to consider structural elements more general than beam-like (slabs, plates, shells, and so on).

Acknowledgements This research was partially supported by: Sapienza Research Grants "Progetti Grandi” 2018 (B81G19000060005); Italian Ministry of University and Research PRIN 2017, project No. 2017HFPKZY (B88D19001130001); Sapienza Research Grants "Progetti Medi" 2017 (Grant No. B83C17001440005, ID: 10.13039/501100004271).

Funding Open access funding provided by Universitá degli Studi di Roma La Sapienza within the CRUICARE Agreement.

Open Access This article is licensed under a Creative Commons Attribution 4.0 International License, which permits use, sharing, adaptation, distribution and reproduction in any medium or format, as long as you give appropriate credit to the original author(s) and the source, provide a link to the Creative Commons licence, and indicate if changes were made. The images or other third party material in this article are included in the article's Creative Commons licence, unless indicated otherwise in a credit line to the material. If material is not included in the article's Creative Commons licence and your intended use is not permitted by statutory regulation or exceeds the permitted use, you will need to obtain permission directly from the copyright holder. To view a copy of this licence, visit http://creativecommons.org/licenses/by/4.0/. 


\section{References}

1. Di Primio, A., Vasta, M., Valente, C., Spina, D.: Monitoring and damage assessment of the Bussi castle vaulted system. In: Proceedings of the International Masonry Society Conferences, vol. 222279, pp. 2356-2366 (2018)

2. Di Primio, A., Fiorini, N., Spina, D., Valente, C., Vasta, M.: Damage assessment of a cloister vault. Lecture Notes in Mechanical Engineering, pp. 314-332 (2020). https://doi.org/10.1007/978-981-138331-1_22

3. Foti, D., Gattulli, V., Potenza, F.: Output-only identification and model updating by dynamic testing in unfavorable conditions of a seismically damaged building. Comput. Aided Civ. Infrastruct. Eng. 29(9), 659-675 (2014). https://doi.org/10.1111/mice.12071

4. Boscato, G., Reccia, E., Baraldi, D., Cecchi, A.: Sensitivity to damage imperfection for multileaf masonry walls based on vibrational analyses. Shock Vib. (2018). https://doi.org/10.1155/2018/ 2321589

5. Cavadas, F., Smith, I., Figueiras, J.: Damage detection using data-driven methods applied to movingload responses. Mech. Syst. Signal Process. 39(1-2), 409-425 (2013). https://doi.org/10.1016/j.ymssp. 2013.02.019

6. Romeo, F., Lofrano, E., Paolone, A.: Damage identification in a parabolic arch via orthogonal empirical mode decomposition. In: Proceedings of the ASME Design Engineering Technical Conference, vol. 8 (2014). https://doi.org/10.1115/DETC2014-35529

7. Gres, S., Ulriksen, M., Döhler, M., Johansen, R., Andersen, P., Damkilde, L., Nielsen, S.: Statistical methods for damage detection applied to civil structures. Procedia Eng. 199, 1919-1924(2017). https:// doi.org/10.1016/j.proeng.2017.09.280

8. Garcia-Perez, A., Amezquita-Sanchez, J., Dominguez-Gonzalez, A., Sedaghati, R., Osornio-Rios, R., Romero-Troncoso, R.: Fused empirical mode decomposition and wavelets for locating combined damage in a truss-type structure through vibration analysis. J. Zhejiang Univ. Sci. A 14(9), 615-630 (2013). https://doi.org/10.1631/jzus.A1300030

9. Lofrano, E., Romeo, F., Paolone, A.: A pseudo-modal structural damage index based on orthogonal empirical mode decomposition. Proc. Inst. Mech. Eng. Part C J. Mech. Eng. Sci. 233(23-24), 75457564 (2019). https://doi.org/10.1177/0954406219885972

10. Lepidi, M., Gattulli, V., Vestroni, F.: Static and dynamic response of elastic suspended cables with damage. Int. J. Solids Struct. 44(25-26), 8194-8212 (2007). https://doi.org/10.1016/j.ijsolstr.2007.06. 009

11. Lepidi, M., Gattulli, V., Vestroni, F.: Damage identification in elastic suspended cables through frequency measurement. J. Vib. Control 15(6), 867-896 (2009). https://doi.org/10.1177/ 1077546308096107

12. Trovalusci, P., Varano, V.: Multifield continuum simulations for damaged materials: a bar with voids. Int. J. Multiscale Comput. Eng. 9(5), 599-608 (2011). https://doi.org/10.1615/IntJMultCompEng. 2011002761

13. Trovalusci, P., Varano, V., Rega, G.: A generalized continuum formulation for composite microcracked materials and wave propagation in a bar. J. Appl. Mech. Trans. ASME (2010). https://doi.org/10.1115/ 1.4001639

14. Settimi, V., Trovalusci, P., Rega, G.: Dynamical properties of a composite microcracked bar based on a generalized continuum formulation. Contin. Mech. Thermodyn. 31(6), 1627-1644 (2019). https:// doi.org/10.1007/s00161-019-00761-7

15. Ostachowicz, W., Soman, R., Malinowski, P.: Optimization of sensor placement for structural health monitoring: a review. Struct. Health Monit. 18(3), 963-988 (2019). https://doi.org/10.1177/ 1475921719825601

16. Arbesser-Rastburg, G., Fuchs-Hanusch, D.: Serious sensor placement-optimal sensor placement as a serious game. Water (Switzerland) (2020). https://doi.org/10.3390/w12010068

17. Yi, T.H., Li, H.N.: Methodology developments in sensor placement for health monitoring of civil infrastructures. Int. J. Distrib. Sens. Netw. (2012). https://doi.org/10.1155/2012/612726

18. Papadimitriou, C., Beck, J., Au, S.K.: Entropy-based optimal sensor location for structural model updating. J. Vib. Control 6(5), 781-800 (2000). https://doi.org/10.1177/107754630000600508 
19. Yuen, K.V., Katafygiotis, L., Papadimitriou, C., Mickleborough, N.: Optimal sensor placement methodology for identification with unmeasured excitation. J. Dyn. Syst. Meas. Control Trans. ASME 123(4), 677-686 (2001). https://doi.org/10.1115/1.1410929

20. Papadimitriou, C.: Optimal sensor placement methodology for parametric identification of structural systems. J. Sound Vib. 278(4-5), 923-947 (2004). https://doi.org/10.1016/j.jsv.2003.10.063

21. Papadimitriou, C.: Pareto optimal sensor locations for structural identification. Comput. Methods Appl. Mech. Eng. 194(12-16), 1655-1673 (2005). https://doi.org/10.1016/j.cma.2004.06.043

22. Friswell, M.I., Mottershead, J.E.: Finite Element Model Updating in Structural Dynamics. Kluwer Academic Publishers, New York (1995)

23. Basseville, M., Benveniste, A., Moustakides, G., Rougee, A.: Optimal sensor location for detecting changes in dynamical behavior. IEEE Trans. Automatic Control 32(12), 1067-1075 (1987). https:// doi.org/10.1109/TAC.1987.1104501

24. Pan, C.T.: On the existence and computation of rank-revealing LU factorizations. Linear Algebra Appl. 316(1-3), 199-222 (2000). https://doi.org/10.1016/S0024-3795(00)00120-8

25. Kammer, D.C.: Optimal sensor placement for modal identification using system-realization methods. J. Guidance Control Dyn. 19(3), 729-731 (1996). https://doi.org/10.2514/3.21688

26. Zhang, J., Maes, K., De Roeck, G., Reynders, E., Papadimitriou, C., Lombaert, G.: Optimal sensor placement for multi-setup modal analysis of structures. J. Sound Vib. 401, 214-232 (2017). https:// doi.org/10.1016/j.jsv.2017.04.041

27. Flynn, E., Todd, M.: A Bayesian approach to optimal sensor placement for structural health monitoring with application to active sensing. Mech. Syst. Signal Process. 24(4), 891-903 (2010). https://doi.org/ 10.1016/j.ymssp.2009.09.003

28. Argyris, C., Papadimitriou, C., Lombaert, G.: Optimal sensor placement for response predictions using local and global methods. In: Conference Proceedings of the Society for Experimental Mechanics Series, pp. 229-236 (2020). https://doi.org/10.1007/978-3-030-12075-7_26

29. Meo, M., Zumpano, G.: On the optimal sensor placement techniques for a bridge structure. Eng. Struct. 27(10), 1488-1497 (2005). https://doi.org/10.1016/j.engstruct.2005.03.015

30. Leyder, C., Dertimanis, V., Frangi, A., Chatzi, E., Lombaert, G.: Optimal sensor placement methods and metrics-comparison and implementation on a timber frame structure. Struct. Infrastruct. Eng. 14(7), 997-1010 (2018). https://doi.org/10.1080/15732479.2018.1438483

31. Maes, K., Lourens, E., Van Nimmen, K., Reynders, E., De Roeck, G., Lombaert, G.: Design of sensor networks for instantaneous inversion of modally reduced order models in structural dynamics. Mech. Syst. Signal Process. 52-53(1), 628-644 (2015). https://doi.org/10.1016/j.ymssp.2014.07.018

32. Guo, H., Zhang, L., Zhang, L., Zhou, J.: Optimal placement of sensors for structural health monitoring using improved genetic algorithms. Smart Mater. Struct. 13(3), 528-534 (2004). https://doi.org/10. 1088/0964-1726/13/3/011

33. Yi, T.H., Li, H.N., Gu, M.: Optimal sensor placement for health monitoring of high-rise structure based on genetic algorithm. Math. Probl. Eng. (2011). https://doi.org/10.1155/2011/395101

34. Sun, H., Büyüköztürk, O.: Optimal sensor placement in structural health monitoring using discrete optimization. Smart Mater. Struct. (2015). https://doi.org/10.1088/0964-1726/24/12/125034

35. He, H., Xu, H., Wang, X., Zhang, X., Fan, S.: Optimal sensor placement for spatial structure based on importance coefficient and randomness. Shock Vib. (2018). https://doi.org/10.1155/2018/7540129

36. Ciambella, J., Pau, A., Vestroni, F.: Effective filtering of modal curvatures for damage identification in beams. Procedia Eng. 199, 1876-1881 (2017). https://doi.org/10.1016/j.proeng.2017.09.119

37. Pingaro, M., Maurelli, G., Venini, P.: Analysis and damage identification of a moderately thick cracked beam using an interdependent locking-free element. J. Optim. Theory Appl. (2020). https://doi.org/ 10.1007/s10957-020-01637-6

38. Lofrano, E., Paolone, A., Vasta, M.: A perturbation approach for the identification of uncertain structures. Int. J. Dyn. Control 4(2), 204-212 (2016). https://doi.org/10.1007/s40435-015-0171-4

39. Lofrano, E., Paolone, A., Ruta, G., Taglioni, A.: Perturbation damage indicators based on complex modes. Procedia Eng. 199, 1949-1954 (2017). https://doi.org/10.1016/j.proeng.2017.09.297

40. Lofrano, E., Paolone, A., Ruta, G.: Dynamic damage identification using complex mode shapes. Struct. Control Health Monit. (2020). https://doi.org/10.1002/stc.2632

41. Lofrano, E., Paolone, A., Vasta, M.: Identification of uncertain vibrating beams through a perturbation approach. ASCE-ASME J. Risk Uncertain. Eng. Syst. Part A Civ. Eng. (2016). https://doi.org/10.1061/ AJRUA6.0000845 
42. Lofrano, E., Paolone, A., Pingaro, M., Trovalusci, P.: Optimal sensors placement for damage detection of beam structures. Lecture Notes in Mechanical Engineering pp. 1498-1511 (2020). https://doi.org/ 10.1007/978-3-030-41057-5_121

Publisher's Note Springer Nature remains neutral with regard to jurisdictional claims in published maps and institutional affiliations. 